8th Alexander Friedmann International Seminar

on Gravitation and Cosmology

International Journal of Modern Physics: Conference Series

Vol. 3 (2011) 417-427

(C) World Scientific Publishing Company

DOI: $10.1142 /$ S2010194511000936

\title{
INITIAL DATA FOR NUMERICAL RELATIVITY: THE USE OF SPECTRAL METHODS
}

\author{
M. A. AlCOFORADO, H. P. DE OLIVEIRA and E. L. RODRIGUES \\ Universidade do Estado do Rio de Janeiro \\ Instituto de Física - Departamento de Fúsica Teórica \\ CEP 20550-013 Rio de Janeiro, RJ, Brazil \\ malcoforado@uerj.br,hp.deoliveira@pq.cnpq.br,elrodrigues@uerj.br
}

Received 15 June 2011

Revised 11 July 2011

\begin{abstract}
The determination of physical initial data is an important task in numerical relativity. In this direction we have applied the Galerkin and collocation methods to solve the Hamiltonian constraints resulting from the Cauchy formulation in the cases of spacetimes containing black holes as described by Ref. 1 . We have shown that a considerable improvement in the accuracy is obtained if the basis functions are chosen such that the boundary conditions are satisfied. We have also introduced a new approach to solve numerically the constraint equations which consists in transforming them into parabolic equations after introducing fictitious diffusion terms. As a consequence, the application of Galerkin or collocation methods produces a dynamical system whose stationary solution corresponds to the initial data.
\end{abstract}

Keywords: Initial data; spectral methods.

PACS numbers: 04.25.D-, 02.70.Hm

\section{Introduction}

Spectral methods such as Galerkin, Collocation, Tau, etc. ${ }^{2}$ belong to the general class of Weighted Residual Methods. ${ }^{3}$ These methods represent an alternative strategy to solve numerically any differential equation for which the solution is approximated by a finite series expansion with respect to a convenient set of analytical functions known as basis functions. As a crucial step the series expansion is such that some quantity, a measure of error or residual, which should be exactly zero is forced to be zero in an approximate sense. As a consequence, any spectral method transforms an evolution partial differential equation into a finite set of ordinary differential equations. We highlight two main features of spectral methods: (i) considerable economy of the computational resources to achieve a given accuracy if compared with the finite difference techniques; (ii) the possibility of selecting a coordinate system adapted to the geometry of the problem under consideration that allows the exact treatment of pseudo-singularities present in the chosen coordinates. 
In this paper we implement an efficient use of the Galerkin method with numerical integration (GNI) and collocation method to integrate elliptic partial differential equations arising from the constraint equations of the $3+1$ formulation. The paper is organized as follows. In Sec. 2 we present briefly the basic equations of the problems we are going to consider, and the numerical scheme using GNI and collocation methods. As a matter of fact, we have followed closely the paper ${ }^{1}$ by Kidder and Finn and treated the same models of initial data. In Sec. 3 the details of the numerical scheme are discussed. The elliptic equations are transformed into nonlinear algebraic equations for the modes of the series expansion or grid values of the function under consideration. We also have introduced a new approach to obtain the solution of these equations, which is based on introducing a fictitious diffusion term into the elliptic equations. As a consequence, we obtain a set of nonlinear ordinary differential equations for the grid values whose stationary solution represents the initial data. Section 4 contains the numerical results and the conclusions are in Sec. 5 .

\section{Basic Equations}

The $3+1$ formulation establishes that the spacetime is foliated by spacelike 3dimensional hypersurfaces characterized by $t\left(x^{\alpha}\right)=$ constant, where $t\left(x^{\alpha}\right)$ is a suitable function that can be identified as the time coordinate. In this way, there are two fundamental quantities for the $3+1$ formalism: the metric $\gamma_{i j}$ and the extrinsic curvature $K_{i j}$ of the 3 -dimensional hypersurfaces. It can be shown that the field equations are divided into the constraint and evolution equations. ${ }^{4,5}$

Contrary to the characteristic formulation ${ }^{6}$ of the Einstein's equations, the initial data cannot be specified arbitrarily, but it must satisfy in vacuum four constraint equations given by,

$$
\begin{aligned}
& { }^{(3)} R+K^{2}-K_{i j} K^{i j}=0, \\
& { }^{(3)} \nabla_{j}\left(K^{i j}-K \gamma^{i j}\right)=0,
\end{aligned}
$$

where all quantities are associated to the 3-dimensional hypersurface, and $K=$ $\gamma_{i j} K^{i j}$. These four equations are known as the Hamiltonian and momentum constraints, respectively.

A very convenient way of dealing with the constraint equations is the YorkLichnerowicz ${ }^{5}$ approach that consists in establishing a conformal transformation of the 3-metric in the form,

$$
\gamma_{i j}=\psi^{4} \bar{\gamma}_{i j}
$$

where $\psi$ is the conformal factor and the metric $\bar{\gamma}_{i j}$ is considered given. Also, the extrinsic curvature is separated into its trace $K$ and its trace-free part $A^{i j}=K^{i j}$ $\frac{1}{3} \gamma^{i j} K$. In this approach, the Hamiltonian constraint becomes,

$$
\bar{\nabla}^{2} \psi-\frac{1}{8} \bar{R} \psi-\frac{1}{12} K^{2} \psi^{5}+\frac{1}{8} \bar{A}_{a b} \bar{A}^{a b} \psi^{-7}=0,
$$


where $\bar{\nabla}^{2}$ and $\bar{R}$ are the Laplace operator and the Ricci scalar associated to $\bar{\gamma}_{i j}$, respectively. Note that the Hamiltonian constraint (1) was transformed into an elliptic equation for the conformal factor $\psi$. Then, by solving this equation the physical metric $\gamma_{i j}$ is reconstructed once the conformal metric $\bar{\gamma}_{i j}$ is given. As pointed out by Kidder and Finn, ${ }^{1}$ for the initial value problems we are to consider the momentum constraints can be solved analytically. ${ }^{5}$ Therefore, as discussed in the next Section, we shall apply the G-NI and collocation methods to solve numerically the Hamiltonian constraint for the conformal factor.

We have followed Kidder and Finn ${ }^{1}$ and selected three basic problems that consist in axisymmetric spacetimes containing a single black hole. The boundary conditions contain important physical information of the initial data and are crucial for the implementation of the numerical method. For the problems we are going to treat, the black hole is described by the Einstein-Rosen bridge such that the spacetime is inversion symmetric through the throat, and also asymptotically flat. These conditions are summarized as:

- Asymptotic flatness,

$$
\lim _{r \rightarrow \infty} \psi(r)=1
$$

- Inversion symmetry,

$$
\left(\frac{\partial \psi}{\partial r}+\frac{\psi}{2 a}\right)_{r=a}=0
$$

- Axisymmetry,

$$
\left(\frac{\partial \psi}{\partial \theta}\right)_{\theta=0, \pi}=0
$$

The first problem is described by Bowen and York ${ }^{7}$ in which the Hamiltonian constraint is given by

$$
\bar{\nabla}^{2} \psi+\frac{3 P^{2}}{4 r^{4}}\left(1-\frac{a^{2}}{r^{2}}\right)^{2} \psi^{-7}=0,
$$

where $P$ is a constant. It can be shown that the exact solution that satisfies the boundary conditions (5)-(7) is

$$
\psi=\left(1+\frac{2 E}{r}+6 \frac{a^{2}}{r^{2}}+\frac{2 a^{2} E}{r^{3}}+\frac{a^{4}}{r^{4}}\right)^{1 / 4},
$$

where $E=\sqrt{P^{2}+4 a^{2}}$ is the ADM energy.

The second problem is to determine the axisymmetric black hole spacetime with angular momentum studied for the first time in Ref. 7, and later analyzed numerically. ${ }^{8,9}$ The Hamiltonian constraint reads,

$$
\bar{\nabla}^{2} \psi+\frac{9 J^{2} \sin ^{2} \theta}{4 r^{6}} \psi^{-7}=0
$$


where $J$ is the angular momentum of the physical space. In these two problems the conformal metric is chosen to be flat.

Finally, as the third problem we have considered a black hole perturbed with a Brill wave. ${ }^{10}$ The initial 3-dimensional hypersurface is an isometry surface, or time symmetric. As a consequence, the extrinsic curvature $K_{i j}$ vanishes and the momentum constraints are satisfied. The conformal background metric is chosen as:

$$
d \bar{s}^{2}=\mathrm{e}^{2 q}\left(d r^{2}+r^{2} d \theta^{2}\right)+r^{2} \sin ^{2} \theta d \phi^{2}
$$

where the function $q$ is given by,

$$
q=A_{0} \sin ^{n} \theta\left[\exp \left[-\left(\frac{\eta+\eta_{0}}{\sigma}\right)^{2}\right]+\exp \left[-\left(\frac{\eta-\eta_{0}}{\sigma}\right)^{2}\right]\right] .
$$

Here $\eta=\ln (r / a), n \geq 2$ is an even integer, and $A_{0}, \eta_{0}$ and $\sigma$ are constants associated to amplitude, position and width, respectively, of the Brill wave. According to Ref. 11, the Hamiltonian constraint turns to be,

$$
\frac{\partial^{2} \psi}{\partial r^{2}}+\frac{2}{r} \frac{\partial \psi}{\partial r}+\frac{1}{r^{2}} \frac{\partial^{2} \psi}{\partial \theta^{2}}+\frac{\cot \theta}{r^{2}} \frac{\partial \psi}{\partial \theta}+\frac{\psi}{4} R=0
$$

where

$$
R=\frac{\partial^{2} q}{\partial r^{2}}+\frac{1}{r} \frac{\partial q}{\partial r}+\frac{1}{r^{2}} \frac{\partial^{2} q}{\partial \theta^{2}}
$$

\section{Numerical Scheme}

As in Refs. 1, 5 it will be convenient to introduce the compacfied variable $x$ given by

$$
x=\frac{2 a}{r}-1,
$$

where $a \leq r<\infty$ is mapped onto $-1 \leq x \leq 1$. The angular variable is also changed according to,

$$
y=\cos \theta,
$$

mapping $0 \leq \theta \leq \pi$ onto $-1 \leq y \leq 1$. With these new variables the Hamiltonian constraints for the first, second and third problems become, respectively,

$$
\begin{aligned}
(1+x)^{2} \frac{\partial^{2} \psi}{\partial x^{2}}+\frac{\partial}{\partial y}\left[\left(1-y^{2}\right) \frac{\partial \psi}{\partial y}\right]+\frac{3 P^{2}}{256 a^{2}}\left(3-2 x-x^{2}\right)^{2} \psi^{-7} & =0 \\
(1+x)^{2} \frac{\partial^{2} \psi}{\partial x^{2}}+\frac{\partial}{\partial y}[ & {\left[\left(1-y^{2}\right) \frac{\partial \psi}{\partial y}\right]+\frac{9 J^{2}}{64 a^{4}}\left(1-y^{2}\right)(1+x)^{4} \psi^{-7}=0 } \\
(1+x)^{2} \frac{\partial^{2} \psi}{\partial x^{2}}+\frac{\partial}{\partial y}\left[\left(1-y^{2}\right) \frac{\partial \psi}{\partial y}\right]+\frac{1}{4} \psi R & =0
\end{aligned}
$$


and the boundary conditions are expressed as

$$
\begin{aligned}
\lim _{x=-1} \psi & =1, \\
\left(\frac{\partial \psi}{\partial x}-\frac{1}{4} \psi\right)_{x=1} & =0 .
\end{aligned}
$$

The condition for axisymmetry is satisfied trivially since $\partial \psi / \partial \theta=-\partial \psi / \partial y \sin \theta$ vanishes in $\theta=0, \pi$.

In Ref. 1, Kidder and Finn adopted Chebyshev polynomials as the basis functions for approximating the function $\psi(x, y)$. We followed the prescription of the Galerkin method by choosing basis functions that fulfill the boundary conditions whenever possible. Then, we propose the following spectral expansion:

$$
\psi_{N}(x, y)=1+\sum_{k=0}^{N_{x}} \sum_{j=0}^{N_{y}} b_{k j} \chi_{k}(x) P_{j}(y)
$$

where the subscript $N$ indicates the approximate function, $P_{j}(y)$ are the Legendre polynomials and $\chi_{k}(x)$ are given by the following combination of Chebyshev polynomials,

$$
\chi_{k}(x)=\frac{1}{2}\left(T_{k+1}(x)+T_{k}(x)\right),
$$

in which $\chi_{k}(-1)=0$, and consequently the boundary condition (20) is automatically satisfied. The boundary condition (21) is enforced by imposing that the modes $b_{k j}$ satisfy the condition,

$$
\left(\frac{\partial \psi_{N}}{\partial x}-\frac{1}{4} \psi_{N}\right)_{x=1, y=y_{k}}=0
$$

where $y_{k}, k=0,1 . ., N_{y}$, are the grid or collocation points to be specified in the sequence.

The substitution of the Galerkin expansion (22) into the Hamiltonian constraints results in the corresponding residual equations. Taking, for instance, Eq. (18) we have

$$
\operatorname{Res}_{H}(x, y)=(1+x)^{2} \frac{\partial^{2} \psi_{N}}{\partial x^{2}}+\frac{\partial}{\partial y}\left[\left(1-y^{2}\right) \frac{\partial \psi_{N}}{\partial y}\right]+\frac{9 J^{2}}{64 a^{4}}\left(1-y^{2}\right)(1+x)^{4} \psi_{N}^{-7}
$$

Here the modes $b_{k j}$ are determined such that the residual vanish in an average sense or in the mean. ${ }^{3}$ This means that the inner products (projections) of the residual equations with respect to the test functions are made equal to zero, or

$$
\left\langle\operatorname{Res}_{H}(x, y), \phi_{m n}(x, y)\right\rangle=\int_{-1}^{1} \int_{-1}^{1} \operatorname{Res}_{H}(x, y) \phi_{m n}(x, y) w(x) v(y) d x d y=0,
$$


where $w(x), v(y)$ are the weights functions, and the choice of the test functions $\phi_{m n}(x, y)$ determines the type of spectral method. The Galerkin method is characterized by selecting $\phi_{m n}(x, y)=\chi_{m}(x) P_{n}(y)$, and according to the G-NI version the above integrals are evaluated using quadrature formulas,

$$
\int_{-1}^{1} \int_{-1}^{1} \operatorname{Res}_{H}(x, y) \chi_{m}(x) P_{n}(y) w(x) v(y) d x d y \approx \sum_{j=0}^{M_{x}} \sum_{k=0}^{M_{y}} \operatorname{Res}_{H}\left(x_{k}, y_{j}\right) w_{k} v_{j}=0,
$$

where $w_{k}, v_{j}$ are the discretized weights, and $x_{k}, y_{j}$ are the collocation or grid points given by,

$$
\begin{aligned}
& x_{k}=\cos \left(\frac{k \pi}{M_{x}}\right), k=0,1, . ., M_{x}, \\
& y_{k}=-1, \text { zeros of } \frac{d P_{M_{y}}}{d x}, 1,
\end{aligned}
$$

which are the Chebyshev-Gauss-Lobatto and Legendre-Gauss-Lobatto points, ${ }^{2,12}$ respectively. In general, we may set $M_{x}>N_{x}, M_{y}>N_{y}$ to evaluate the integrals (27) with better accuracy.

The collocation method is characterized by choosing the test functions as Dirac delta functions, $\phi_{m n}(x, y)=\delta\left(x-x_{m}\right) \delta\left(y-y_{n}\right)$, where $x_{m}, y_{n}$ are collocation points given above for which $M_{x}=N_{x}, M_{y}=N_{y}$. The vanishing of the inner products implies that the residual equation is forced to be zero exactly at the collocation points,

$$
\operatorname{Res}_{H}\left(x_{m}, y_{n}\right)=0
$$

with $m=0,1, . ., N_{x}$ and $n=0,1, . ., N_{y}$.

The resulting Eqs. (27) or (29) constitute sets of nonlinear algebraic equations for the modes $b_{k j}$, or may also be expressed in terms of the grid values $\psi_{k j}=\psi_{N}\left(x_{k}, y_{j}\right)$. Once the solution is obtained the approximate initial data function $\psi_{N}(x, y)$ is reconstructed. However, a nonlinear algebraic system does not possess a unique solution and the determination of the correct solution can be a difficult task. In order to circumvent this problem, we have added a fictitious diffusion term ${ }^{13}$ in the Hamiltonian constraint in order to deal with a set of ordinary differential equations for the grid values $\psi_{k j}$ instead a set of nonlinear algebraic equations. For instance, with respect to the second problem, the residual equation (18) with the fictitious diffusion-like term is,

$$
\begin{aligned}
\operatorname{Res}_{H}(t, x, y) & =-\frac{\partial \psi_{N}}{\partial t}+(1+x)^{2} \frac{\partial^{2} \psi_{N}}{\partial x^{2}}+\frac{\partial}{\partial y}\left[\left(1-y^{2}\right) \frac{\partial \psi_{N}}{\partial y}\right] \\
& +\frac{9 J^{2}}{64 a^{4}}\left(1-y^{2}\right)(1+x)^{4} \psi_{N}^{-7}
\end{aligned}
$$

After the projections we obtain the set of equations $(\partial \psi / \partial t)_{k j}=F_{k j}\left(\psi_{l m}\right)$. The result of the projections (27) or (29) becomes a nonlinear dynamical system for 
the grid values $(\partial \psi / \partial t)_{k j}$. The correct solution of the Hamiltonian constraint is a stationary solution of the generated dynamical system, which can be determined numerically.

\section{Numerical Results}

We present here the results obtained by solving the Hamiltonian constraint using G-NI and collocation methods. In order to certify the accuracy and convergence of the results we have proceeded as follows:

- In the first problem we have compared the exact and the numerical solutions. The error measure is the $L_{2}$ norm,

$$
L_{2}=\left(\frac{1}{2} \int_{-1}^{1}\left(\psi_{\operatorname{exact}(x)}-\psi_{N}(x)\right)^{2} d x\right)^{1 / 2},
$$

where $\psi_{\text {exact }}$ is given by Eq. (9).

- For any other problem two tests were established. The first is to investigate if the residual equation associated to the Hamiltonian constraint is sufficiently small and tends to zero with the increase of the truncation orders. A good measure of the error is following $L_{2}$ norm given by,

$$
L_{2}(\operatorname{Res})=\left(\frac{1}{4} \int_{-1}^{1} \int_{-1}^{1} \operatorname{Res}_{H}(x, y)^{2} d x d y\right)^{1 / 2} .
$$

Another valid measure of the accuracy and convergence of the numerical scheme is the $L_{2}$ norm associated to the difference between two successive approximate solutions, or $L_{2}(\delta \psi)$, where $\delta \psi=\psi_{N_{x}}-\psi_{N_{x}-2}$.

In Fig. 1 we show the plots of the $L_{2}$ norm (31) evaluated for several truncation orders $N_{x}$. The graph on the left was obtained applying the G-NI method, whereas
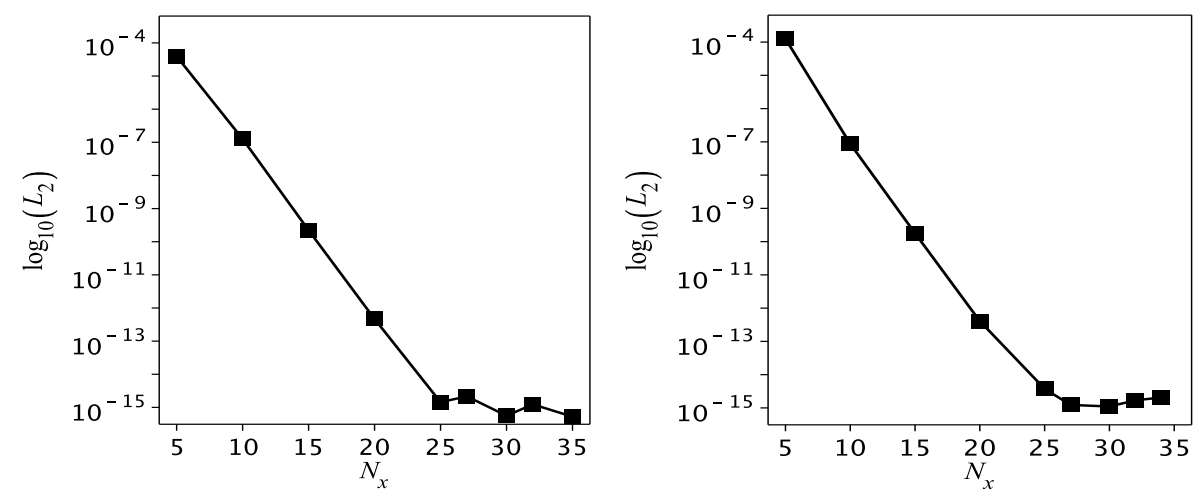

Fig. 1. Decay of the error between the exact and approximate solutions expressed by the $L_{2}$ norm (31) with the increase of the truncation order $N_{x}$. We have set $P=1, a=1$. The graph on left was obtained with the G-NI method in which $M_{x}=1.5 N_{x}$, whereas in the graph on right we have used the collocation method. In both cases the exponential decay of the $L_{2}$ norm is observed until the saturation due to the round off error. 


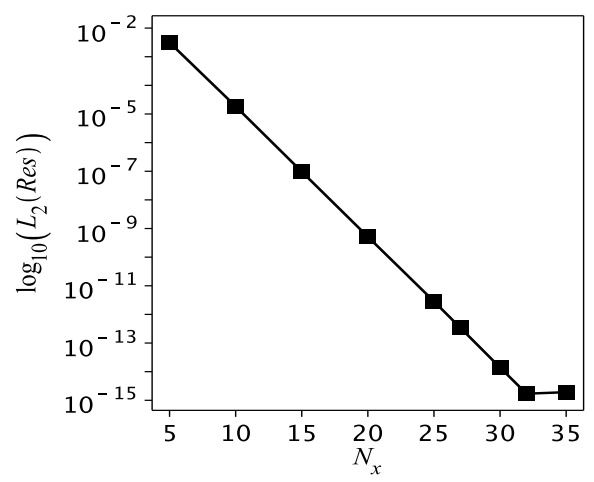

Fig. 2. Decay of the norm of the residual equation associated to Eq. (17). The saturation occurs for $N_{x}=30$.

the graph on the right with the collocation method. Note that in both cases the typical spectral convergence with the exponential decrease of the error when the truncation order increases. Although the G-NI method appears to be slightly more accurate than the collocation method, its implementation is usually more costly due to the evaluation of the integrals. In both cases the saturation of $L_{2}$ due to round-off error in about $10^{-14}$ is achieved for $N_{x}=25$. As a matter of fact, this result is better than the similar found by Kidder and Finn ${ }^{1}$ using pseudospectral method for which the basis functions are pure Chebyshev polynomials. In their case, for $N_{x}=30 L_{2} \sim \mathcal{O}\left(10^{-13}\right)$. The decay of $L_{2}$ (Res) is shown in Fig. 2, where the saturation due to round-off error is found for $N_{x} \geq 30$. In all cases $P=1$ and $a=1$.

The second problem is an axisymmetrical problem of a rotating black hole with equatorial plane symmetry, implying that $\psi$ is even in $y$. Since no exact solution is available, the accuracy and convergence tests were performed by evaluating the norms $L_{2}$ (Res) and $L_{2}(\delta \psi)$. The set of nonlinear equations (29) was solved numerically for the modes $b_{k j}$. However, for large truncation orders we have considered the dynamical system approach for determining the grid values $\psi_{l m}$ [cf. Eqs. (29) and (30)]. The results of both measures of error are shown in Fig. 3 with $J=1$ and $a=1$. $N_{y}$ was kept fixed $\left(N_{y}=8\right)$ and $N_{x}$ varies from $N_{x}=8$ to $N_{x}=26$ producing a rapid convergence until the saturation due to round-off error is achieved. It must be noticed that $L_{2}(\delta \psi) \approx 10^{-13}$ for $N_{y}=20$, while to obtain the same value Kidder and Finn ${ }^{1}$ used $N_{x}=30$.

The last problem is described by the Hamiltonian constraint (19) with $R$ given by Eq. (14) and the function $q(x, y)$ specified by expression (12). As in Ref. 1 the following values of the Brill wave parameters were considered: $\sigma=1, \eta_{0}=A_{0}=1$ and $n=2$. We present in Fig. 4(a) the error $L_{2}(R)$ after applying the collocation method, in which the approximate function $R$ is given by,

$$
R_{a}(x, y)=\sum_{k=0}^{N_{x}} \sum_{j=0}^{N_{y}} c_{k j} \chi_{k}(x) T_{j}(y)
$$



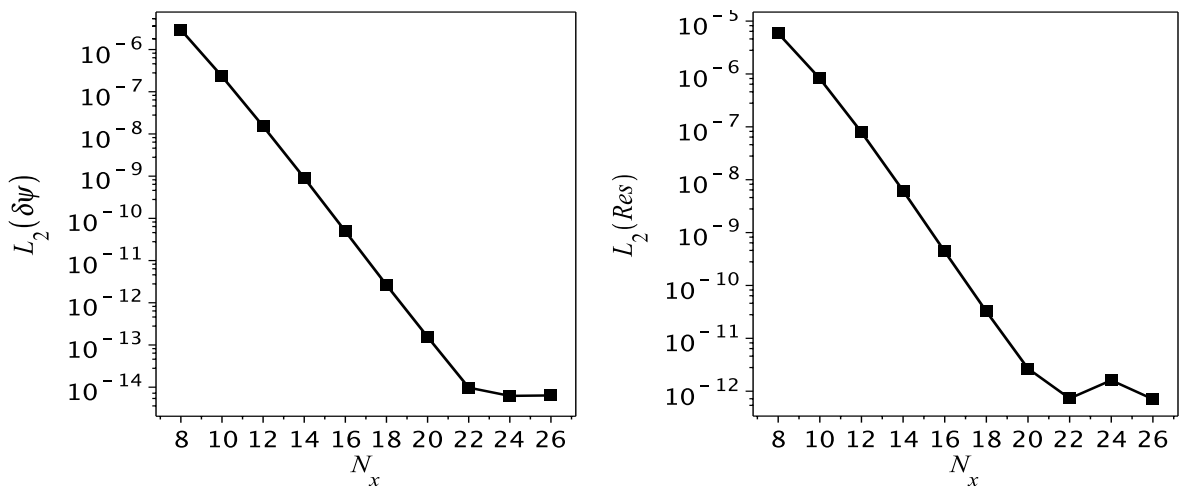

Fig. 3. Exponential decay of $L_{2}(\delta \psi)$ and $L_{2}$ (Res) for second problem with $J=1, a=1$. Here $N_{y}=8$ is fixed and $\delta \psi=\psi_{N_{x}}-\psi_{N_{x}-2}$.

The basis functions $\chi_{k}(x), P_{j}(y)$ satisfy the appropriate boundary conditions $^{10}$ for $R$. We have fixed $N_{y}=8$ and evaluated the error for distinct values of $N_{x}$ [cf. Fig. 4(a)]. Comparing the plot of Fig. 4(a) with Fig. 5 of Ref. 1, it is clear the improvement in the accuracy by taking the approximation (33). For instance, according to Fig. 4(a) to obtain $L_{2}(R) \sim \mathcal{O}\left(10^{-7}\right)$ we need $N_{x}=50$, whereas in Ref. 1 the same error is achieved with $N_{x}=65$.

The last numerical experiment is the verification of the error in the residual equation associated to the Hamiltonian constraint, where we have considered the exact expression for $R$ given by (14). The decay of the norm $L_{2}(R)$ as shown in Fig. 4 (b) is nearly exponential with an approximate periodic component.

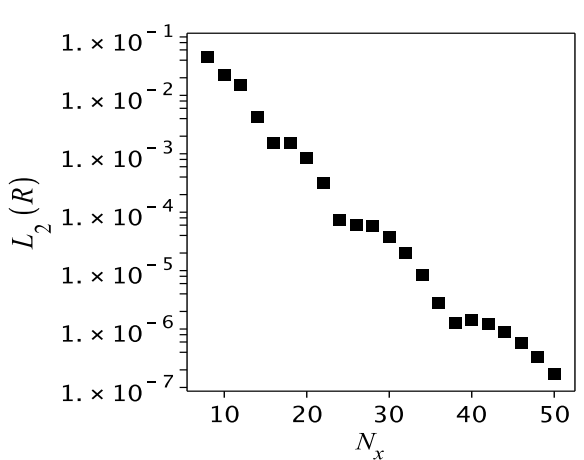

(a)

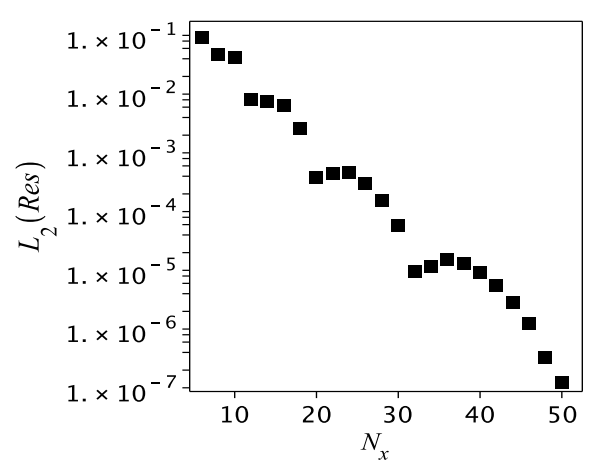

(b)

Fig. 4. Decay of the norms $L_{2}(R)$ and $L_{2}$ (Res) for the problem of a black hole perturbed by a Brill wave. In both cases $A_{0}=\eta_{0}=\sigma=1, N y=8$ is fixed, and $N_{x}$ varies in the interval $N_{x}=6, . ., 50$. 


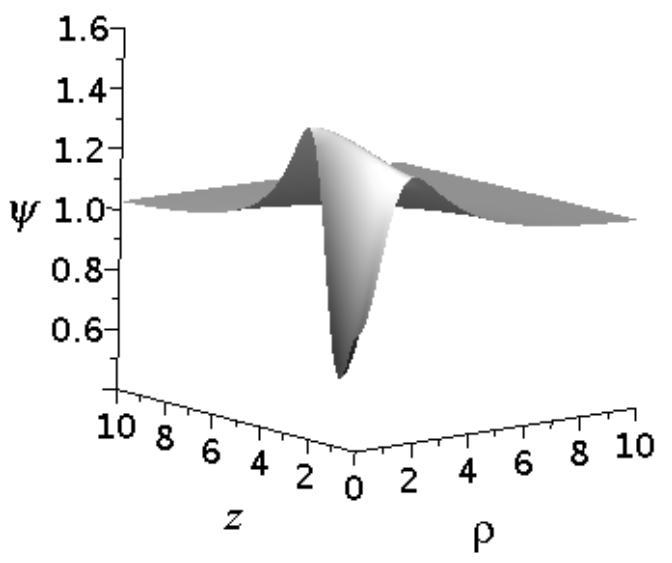

Fig. 5. Conformal factor $\psi(\rho, z)$ for the Brill wave initial data with $q(\rho, z)=A_{0} \rho^{2} \mathrm{e}^{-\left(\rho^{2}+z^{2}\right)}$, with $A_{0}=10$. We have considered $N_{\rho}=30, N_{z}=10$.

\section{Further Perspectives}

In this paper we have solved the Hamiltonian constraint to determine the initial data for several axisymmetric spacetimes containing a black hole. This equation is a nonlinear elliptical partial differential equation, whose approximate solution for each case of interest was obtained using the G-NI and collocation methods. These problems have been already addressed by Kidder and Finn ${ }^{1}$ in the context of the pseudospectral method. The novelty of the present treatment was the use of basis functions in which each element satisfies most of the boundary conditions, which is a basic requirement for the Galerkin method. As a consequence, our numerical results shown to be more accurate than corresponding results obtained using pseudospectral method. ${ }^{1}$ We have also presented a new approach to solve numerically the elliptic constraint equation by transforming it into a parabolic equation by introducing a fictitious diffusion term [see Eq. (30)]. The application of the spectral method produces a dynamical system whose stationary solution is the solution of the corresponding elliptic equation.

Motivated by the improvement in the accuracy when a relatively simple change of the basis functions is done, our next step will be to evolve pure Brill ${ }^{10}$ waves. In this case the first step is to solve the constraint equation (13) to provide the initial data for the evolution equations. Since there is no black hole the radial domain is $0 \leq r<\infty$, and the choice of the $q(x, y)$ is distinct of the one given by (12). In Fig. 5 we present the approximate conformal factor $\psi(\rho, z)$ for $q=A_{0} \rho^{2} \mathrm{e}^{-\left(\rho^{2}+z^{2}\right) 14}$ with 
$A_{0}=10$, where $\rho, z$ are cylindrical coordinates. In this case we have considered

$$
\psi_{N}(\rho, z)=1+\sum_{k, j=0}^{N_{\rho}, N_{z}} b_{k j} \chi_{k}(\rho) \phi_{j}(z),
$$

where the basis functions $\chi_{k}(\rho), \phi_{j}(z)$ satisfy the boundary conditions $\lim _{r \rightarrow \infty} \psi=1$ $\left(r^{2}=\rho^{2}+z^{2}\right)$ and $(\partial \psi / \partial \rho)_{\rho=0}=0$.

\section{Acknowledgments}

The authors acknowledge the financial support of the Brazilian agencies CNPq, CAPES and FAPERJ.

\section{References}

1. L. Kidder and L. S. Finn, Phys. Rev. D 62, 084026 (2000).

2. P. Grandclément and J. Novak, Living Rev. Relativity 12, 1 (2009); http://www. livingreviews.org/lrr-2009-1; P. Holmes, J. L. Lumley and G. Berkooz, Turbulence, Coherent Structures, Dynamical Systems and Symmetry (Cambridge University Press, Cambridge, 1998); B. Fornberg, A Pratical Guide to Pseudospectral Methods (Cambridge University Press, Cambridge, 1998).

3. B. A. Finlayson, The Method of Weighted Residuals and Variational Principles (Academic Press, New York, 1972).

4. R. Arnowitt, S. Deser and C. W. Misner, The dynamics of general relativity, in Gravitation: An Introduction to Current Research, ed. L. Witten (Wiley, New York, 1962), p.227.

5. J. W. York Jr., The initial value problem and dynamics, in Gravitational Radiation, eds. N. Deruelle and T. Piran (North-Holland, Amsterdan, 1983).

6. J. Winicour, Liv. Rev. Relativity 12, 3 (2009); http://www.livingreviews.org/lrr-20093.

7. J. M. Bowen and J. W. York Jr., Phys. Rev. D 21, 2047 (1980).

8. M. Choptuik and W. G. Unrhuh, Gen. Rel. Gravit. 18, 813 (1986).

9. G. Cook, Liv. Rev. Relativity 3, 5 (2000); http://www.livingreviews.org/lrr-2000-5.

10. D. Brill, Ann. Phys. (N.Y.) 7, 466 (1959).

11. D. Bernstein, D. Hobill, E. Seidel and L. Smarr, Phys. Rev. D 50, 3760 (1994).

12. J. P. Boyd, Chebyshev and Fourier Spectral Methods (Dover Publications, New York, 2001).

13. D. Bazeia, E. da Hora, R. Menezes, H. P. de Oliveira and C. dos Santos, Phys. Rev. D 81, 125016 (2010).

14. D. Holz, W. Miller, M. Wakano and J. Wheeler, Coalescence of primal gravity waves to make cosmological mass without matter, in Directions in General Relativity: Proceedings of the 1993 International Symposium, Maryland; Papers in honor of Dieter Brill, eds. B. L. Hu and T. A. Jacobson (Cambridge University Press, Cambridge, 1993), p. 993. 